\title{
INTERMEDIATE HE RICH STARS
}

\author{
K. HUNGER ${ }^{1)}$, D. GROOTE ${ }^{2)}$, U. HEBER ${ }^{1)}$ \\ 1) Institut für Theoretische Plhysik \\ und Sternwarte der Univ. Kiel \\ Olshausenstr. 40, W-2300 Kiel \\ Germany \\ ${ }^{2)}$ Hamburger Sternwarte \\ Gojenbergweg 112 \\ $W-2050$ Hamburg 80 \\ Germany
}

\section{GENERAL PROPERTIES}

Intermediate helium stars are defined by the number ratio of helium to hydrogen:

$$
0.3<\mathrm{n}_{\mathrm{He}} / \mathrm{n}_{\mathrm{H}}<10
$$

Whereas at the helium rich end, there is a distinct gap between the intermediates and the extreme helium stars, the borderline between the intermediates and the normal B-stars appears less well defined. 24 stars are listed by Drilling and Hill (1986), with $\mathrm{V}<11^{\mathrm{m}}$. It can be anticipated that a major fraction has as yet escaped detection.

The spectral types center around $B 2 \mathrm{~V}$, effective temperatures lie in a rather narow range, $20000 \mathrm{~K}<\mathrm{T}_{\text {eff }}<25000 \mathrm{~K}$, and gravities, so far analyzed, appear to be reduced by up to a factor 2, with respect to the zero age main sequence. Since only one star (HD 37017) is known to be a binary, masses can be determined solely spectroscopically, from $g$ and $T_{\text {eff }}$ if either the distance is known or the state of evolution. In the latter case the mass is read off the $\left(\mathrm{g}, \mathrm{T}_{\text {eff }}\right)$-diagram: $M=6-10 \mathrm{M}_{\odot}$, which again places the intermediate helium stars near the main sequence. With these fundamental stellar parameters, the luminosity is typically $\log \mathrm{L} / \mathrm{L}_{\odot}=3.8$ (HD 37479).

Metals appear (nearly) solar as Bohlender (1988) concludes from the analysis of 5 stars. (In case of variability, the abundances are phase averaged.) Projected rotational velocities also appear distributed as in normal B stars, with 5 stars having $\mathrm{v} \sin \mathrm{i} \approx 150 \mathrm{kms}^{-1}$ and with 3 stars having $\mathrm{v} \sin \mathrm{i}<30 \mathrm{kms}^{-1}$.

Remarkable are the strong magnetic fields, observed in 9 stars. In most cases they can more or less be approximated by (decentered) dipoles, except for the case of HD 37776, where the dominant configuration is that of a quadrupole. The maximum fields observed are $B_{e} \max =3.4 \mathrm{kG}(\delta$ Ori $C)$ and $\mathrm{Bs}_{\max }=58.0 \mathrm{kG}$ (HD 37776). $\mathrm{B}_{\mathrm{pol}}$ is typically $10 \mathrm{kG}$. These are the strongest fields observed in non-degenerates.

Another peculiarity is the variability that occurs in 7 stars. Variability comprises He-absorption, $\mathrm{H} \alpha$-emission, shell absorption, (radial velocity), photometry, magnetic field and polarization, which occur in a given star with one common period. The periods range from $P=0.9$ to 1.7 days, except for HD $184927(P=9.5 d)$. Since moreover the $(P, v \sin i)$ relation is obeyed (with $\left.R>4 R_{\odot}\right)$, the stars are considered as classical oblique rotators. 
Circumstellar matter becomes manifest through radio-emission (HD 37017, HD 37479, $\delta$ Ori C), through $\mathrm{H} \alpha$-emission (5 stars), X-rays (HD 37017, HD 37479) and wind. For HD 37479, Hamann (1981) derived by means of the comoving frame method $M=10^{-10}-10^{-9} M_{\odot} /$ year, and a blue edge velocity of $600 \mathrm{kms}^{-1}$.

The galactic distribution is confined to $\mid \mathrm{bi}<19^{\circ}$, with a major fraction located in the Orion OB1 association (4 stars) and one member of IC 2944.

Evolution: From the foregoing properties it is obvious that helium enrichment is a product of diffusion rather than of evolution. For in the latter case the sensitive number ratio N/C should be larger than 1, as is observed only in two stars HD 64740 (Kaufmann and Schacht, 1975) and HD 186205 (Lester, 1972), whereas in the former case, it should be less than unity (as observed in at least 3 stars). These analyses eventually have to be repeated, with higher $\mathrm{S} / \mathrm{N}$ spectrograms.

The foregoing statistical review is based on the following publications: Hunger 1986a and b (includes bibliography until 1986), Barker 1986, Bohlender et al. 1987, Drake et al. 1987, Philips and Lestrade 1988, Bohlender and Landstreet 1988, Hunger et al. 1989, Bohlender Thesis 1988, Hunger et al. 1990.

\section{THE SPECIAL CASE OF HD 37479}

The remainder of the review is devoted to HD 37479 (formerly $\sigma$ Ori E), which though may not be considered the prototype of its class, but which is the best studied object. It exhibits all the variable features listed in Sect. 1, with the common period $P=1.19081 \mathrm{~d}$. The model that fits the observations reasonably well, is reproduced in Fig. 1 (which is an update of the model proposed by Groote and Hunger, 1982). The axis of rotation is vertical, the magnetic poles are marked by + or - . The shaded areas show the He-caps. The two corotating clouds are centered at the intersection of the magnetic and rotational equators. The clouds extend up to 6 stellar radii. Phases are shown at the periphery (arabic numbers). Roman numbers designate the phases which have been used in the analysis. In the following, general problems which beset the analysis of He-variable stars are briefly discussed.

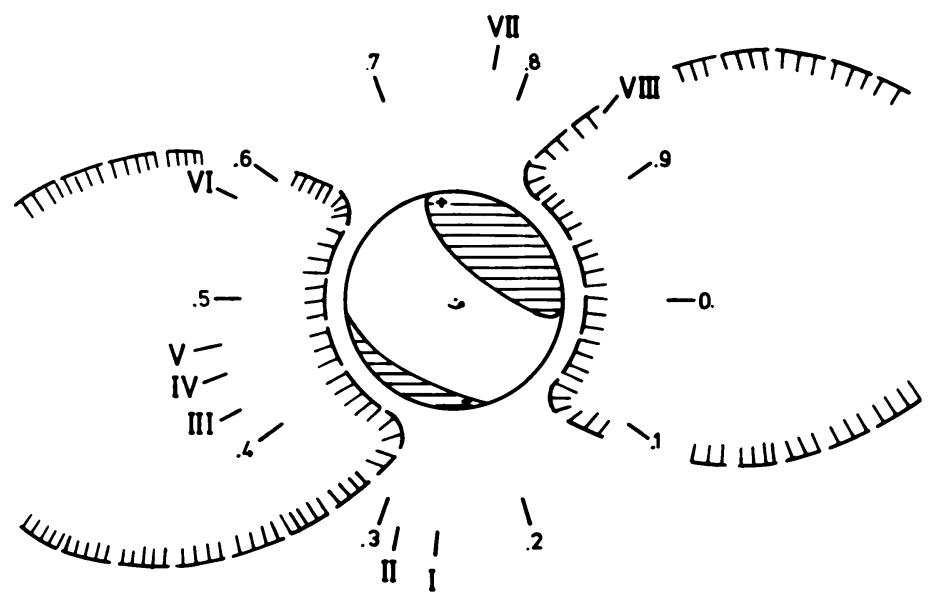

Fig. 1: Updated model of HD 37479 showing the He-patches (shaded), the clouds and the magnetic poles ( + and -$)$. Arabic numbers designate phases, roman numbers those phases for which ESO-CASPEC spectrograms have been used. 


\section{SPECTRAL ANALYSIS AND ITS PROBLEMS}

As a result of rotation $\left(v \sin i=150 \mathrm{kms}^{-1}\right)$ faint lines are washed out, which result in a loss of important abundance informations, and which also render the Doppler imaging of metal spots (almost) impossible. Doppler imaging is likewise not applicable in the case of He-surface mapping, as the strong He lines are saturated.

When attempting to model the surface of a variable Helium star, one must be aware that the simple geometry of 2 symmetric and circular caps may be too schematic. Furthermore, there may exist horizontal and/or vertical abundance gradients. In order to model the Helium surface distribution it is important to have a model atmosphere program that reproduces the line cores accurately and that uses the appropriate phase dependent He-abundances. The Kiel Group uses Kurucz opacity distribution functions and surface averaged He-opacities, while the Western Ontario Group works with standard composition model atmospheres, however uses a refined line synthesis program that calculates the integrated intensity and polarization profiles for the assumed model atmosphere, magnetic geometry, and surface abundance distribution. The Helium maps produced by these groups in fact do show differences. (The best approach would be a combination of the Kiel atmosphere and the Western Ontario line synthesis program.)

The biggest problem poses the contamination of the spectrum by the clouds, with their emission in quadrature and absorption in conjunction. Hunger et al. (1990) have shown that the effect of the clouds can largely be eliminated if proper phases are choosen and the analysis is performed strictly differentially with respect to suitable standards $(\gamma \mathrm{Peg}, \sigma$ Ori $\mathrm{D})$. From the afore mentioned problems, it is clear that we are still far from deriving unambiguous surface maps of helium and metals.

\section{SURFACE DISTRIBUTION OF HELIUM AND METALS.}

The foregoing analysis yields $2 \mathrm{He}$-caps with $\epsilon_{\mathrm{He}}=0.8$, while the rest is close to normal $\left(\epsilon_{\mathrm{He}}=0.15\right)$. The geometry is described by $i=46^{\circ}, \beta=73^{\circ}$ and the cap radius $r=47^{\circ}$ (both caps). No vertical abundance stratification is needed in order to explain the profiles of the strong He-lines. (In essence the same model is proposed by Bohlender, 1989.)

The metals, as a rule, seem to vary in antiphase. The question arises whether there are phases where the metal abundances are solar. This can be answered simply by dividing the (optical) spectrum of HD 34749 by the (rotated) spectrum of $\gamma$ Peg. In those phases where the abundances agree, a straight "continuum" should result. In Fig. 2 a portion of the spectrum near CII is shown. The straight continuum is seen at $\phi=0.57$, a phase where only a small fraction of the He-caps and the polar regions is visible. Inside the He-caps, the metals are depleted by a factor of 10 , by numbers.

In Fig. 3 the variations of the UV equivalent widths are shown, and also of the $\mathrm{H}$-abundance, $\epsilon_{\mathrm{H}}$. The metals exhibit a symmetry axis which is aligned with the magnetic axis which becomes clearly evident at the south pole (marked S). For the north pole a quantitative analysis is needed as at that phase the $\mathrm{H} / \mathrm{He}$ ratio varies drastically. The hydrogen symmetry axis seems to be displaced by $\Delta \phi \approx 0.1$. If proved to be true, the two symmetry axes could mean two different mechanisms for diffusion?!

The results of this section, though still preliminary, can be summarized: diffusion is confined to the He-caps which are located near (or off set by $\approx 45^{\circ}$ ) the intersection of the magnetic and rotational equator (Bohlender, 1988; Hunger et al. 1990). From this fact one is forced to conclude that diffusion is influenced not only by the magnetic field but also by rotation, either by centrifugal forces (as proposed by Bohlender) which reduce gravity and speed up the wind, or by Coriolis forces (as proposed by Hunger et al.) which act as "angular momentum barriers" that brake the wind. In any case, the segregation of chemical element appears to occur in the wind or the bottom of it. 


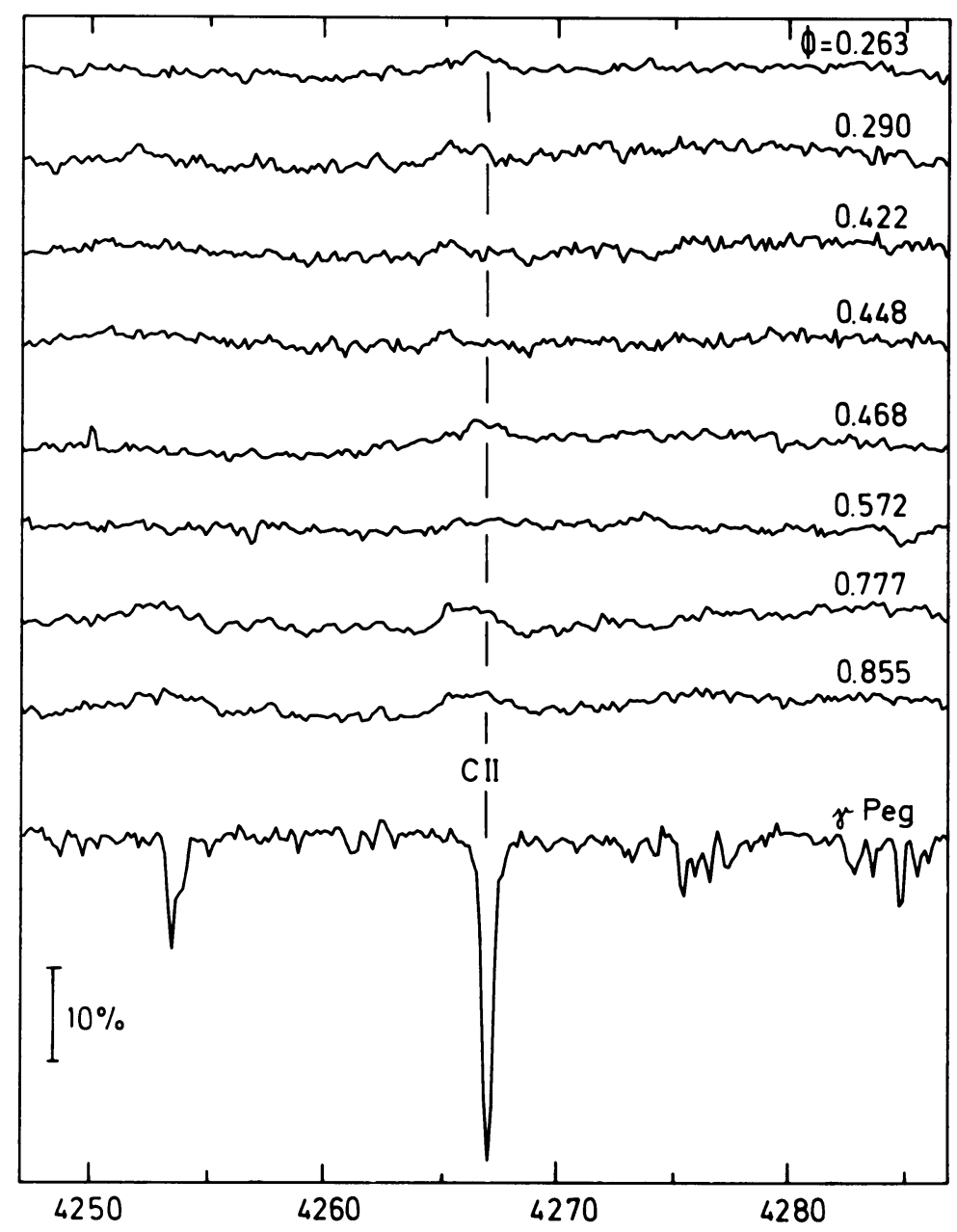

Fig. 2: $\quad$ ESO-CASPEC spectrum centered at $\lambda 4267$ (CII), of the comparison star $\gamma$ Peg (bottom), and the normalized spectrum of HD 37479 which is divided by the (rotationally broadened) spectrum of $\gamma$ Peg. The phases increase from top to bottom. 


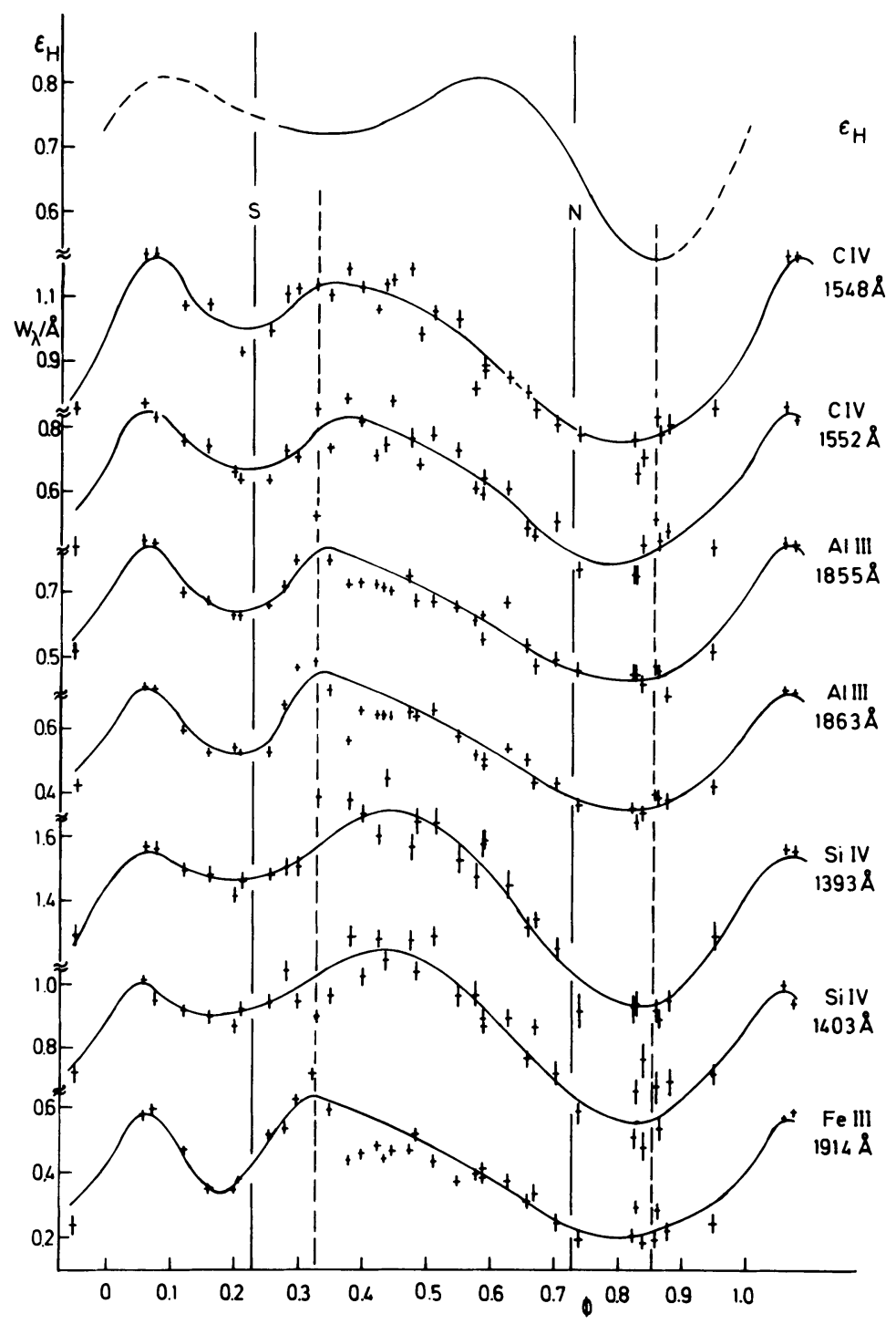

Fig. 3: Phase variations of UV metal line equivalent widths and of the hydrogen number fraction (top). 


\section{THE WIND AND THE ANGULAR MOMENTUM BARRIER.}

The mass loss from HD 37479 is estimated to $\dot{\mathrm{M}}=10^{-10}-10^{-9} \mathrm{M}_{\odot} / \mathrm{yr}$ (Hamann, 1981). The theory of radiatively driven winds predicts $M \approx 410^{-9} \mathrm{M}_{\odot} / \mathrm{yr}$ (Pauldrach, 1989). $90 \%$ of the wind is trapped in the magnetosphere (clouds), which extends equatorially up to $L=6 R_{*}$, see Fig. 4. (From $L=6 R_{*}$, a polar cone with $\theta_{0}=24^{\circ}$ results.) $10 \%$ of the wind excapes freely, in agreement with the observations.

The bound part of the wind is further restricted, as can be demonstrated on hand of Fig. 5. The radiation driven wind reaches its "terminal" velocity $\left(\approx 600 \mathrm{kms}^{-1}\right)$ already at some $1.2 R_{4}$. Beyond this point the wind particles move more or less freely under the influence of gravitation, if the magnetic field is zero. In a corotating frame, trajectories develop which are shown for the first and fourth quadrant, as full drawn curves and labelled by their "terminal" velocities. In case of a (dipole) magnetic field, there is little interaction with the field as long as the trajectories do not cross magnetic field lines (dashed lines). This is the case for the first quadrant and velocities in the range of $400-1200 \mathrm{kms}^{-1}$. The wind particles are able to reach the outer part of the magnetosphere. This is not the case for the fourth quadrant: wind ions cross the field lines, are trapped and do not reach the outer zones. This picture is admittedly crude, but it makes plausible that the wind feeding the clouds comes predominantly from the first and third quadrant. It is important to note that the two quadrants are essentially identical with the He-caps. From this picture one is tempted to believe in a correlation between Helium enrichment and the wind. The next step then is to ask whether the He-enrichment is caused by the wind.

\section{THE HAVNES AND GOERTZ MECHANISM.}

The mass of clouds is estimated by Groote and Hunger (1982): $\mathrm{M} \approx 10^{-10} \mathrm{M}_{\odot}$. The mass is replenished by the wind at the rate of $10^{-9} \mathrm{M}_{\odot} / \mathrm{yr}$, i.e. once per month. At the same rate, the clouds loose mass, through field line reconnections as is sketched in Fig. 6. Thereby large amounts of energy are released. The Havnes and Goertz theory (1984) predicts an outer magnetosphere with a corona of $T=10^{6}$. $10^{7} \mathrm{~K}$, and the production of mildly relativistic electrons, in the MeV-range. The observations seem to confirm this: X-rays are observed, with $\log \left(\mathrm{L}_{\mathrm{x}} / \mathrm{L}_{\odot}\right)=3.8$, and radio emission at 2 and $6 \mathrm{~cm}$, indicating a (non-thermal) flat spectrum (Drake et al. 1987). Philips and Lestrade, using the spatial resolution of VLBI, were able to locate the emission zone, which extends from 6 to $10 R_{\star}$ (based on the new distance of HD 37479, $d=640 \mathrm{pc}$, Hunger et al. 1989).

In Fig. 7, a model of the magnetosphere is shown, which seems to apply to HD 37479, with the exception that in our star the axis of rotation is (almost) vertical. As a consequence, the plasma sheet is forced to trail in an Archimedic spiral. It is interesting to note that this model has been proposed for the infrared source S1, in the $\rho$ Oph molecular cloud (André et al. 1988). S1 is believed to be a pre main sequence object. HD 37479 and S1 have in common (probably) the early spectral type, the radioand $\mathrm{X}$-ray emission, and the magnetic field (indicated by the polarized radio emission). If the similarity is further corroborated, it could mean that all magnetic and variable He-stars are pre main sequence stars.

According to the tracks of Iben (1966), the mass of HD 37479 would be $8 \mathrm{M}_{\odot}$, instead of $8.9 \mathrm{M}_{\odot}$ as a post main sequence star. The birth line of stars (Paller and Stahler, 1990), however, reaches the main sequence for stars with $M>10 \mathrm{M}_{\odot}$. Therefore one would expect a substantial amount of still unaccreted matter around a recently born star with $M=8 \mathrm{M}_{\odot}$. The question then arises whether the circumstellar matter around HD 37479 comes from the wind or is the unaccreted rest of the protostellar nebula. 


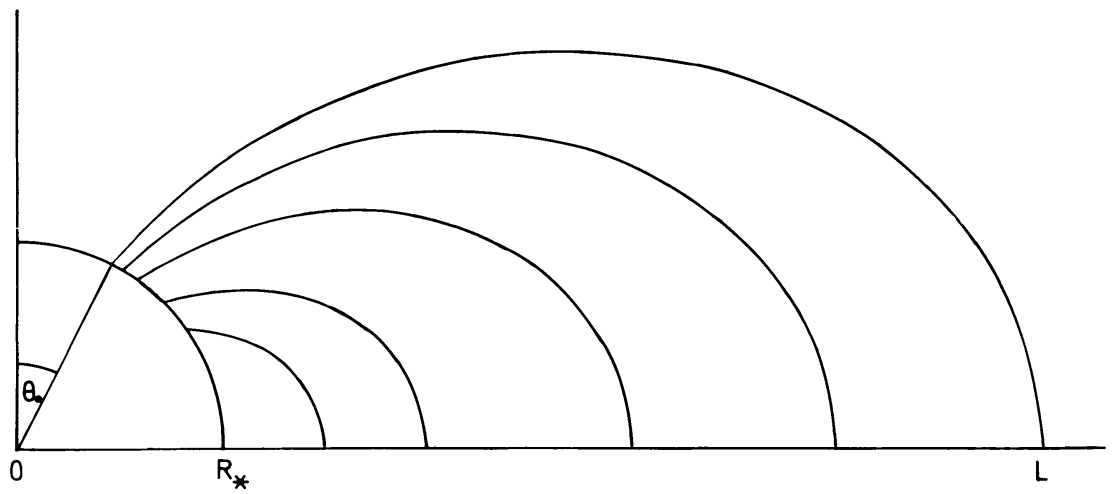

Fig. 4: Magnetic dipole field of HD 37479. Since the clouds extend up to $6 R_{w}$ field lines are assumed to be open within the polar cone $\left(\theta_{0}=24^{\circ}\right)$.

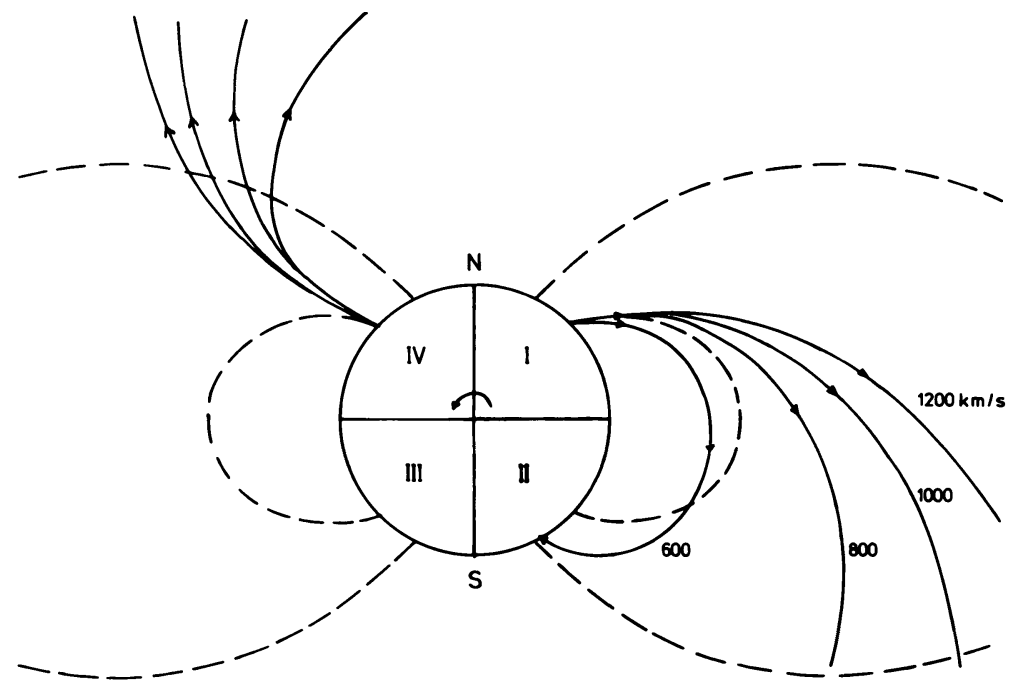

Fig. 5: Trajectories of particles moving in a rotating frame under the influence of gravity, for various injection velocities (full drawn). Dashed curves represent two magnetic lines of forces. In the first quadrant, particle trajectories are nearly parallel to the field lines (no interaction with the field), whereas in the fourth quadrant, trajectories cross the field lines (interaction with the field). 


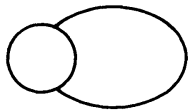

Fig. 6: Schematic drawing of how the stellar

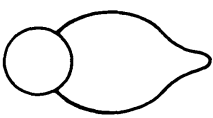
wind of HD 37479 gradually fills the magnetosphere (clouds) until the field lines are distorted and reconnect, thereby releasing large amounts of energy (after Havnes and

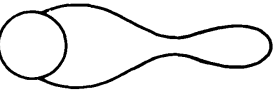
Goertz).
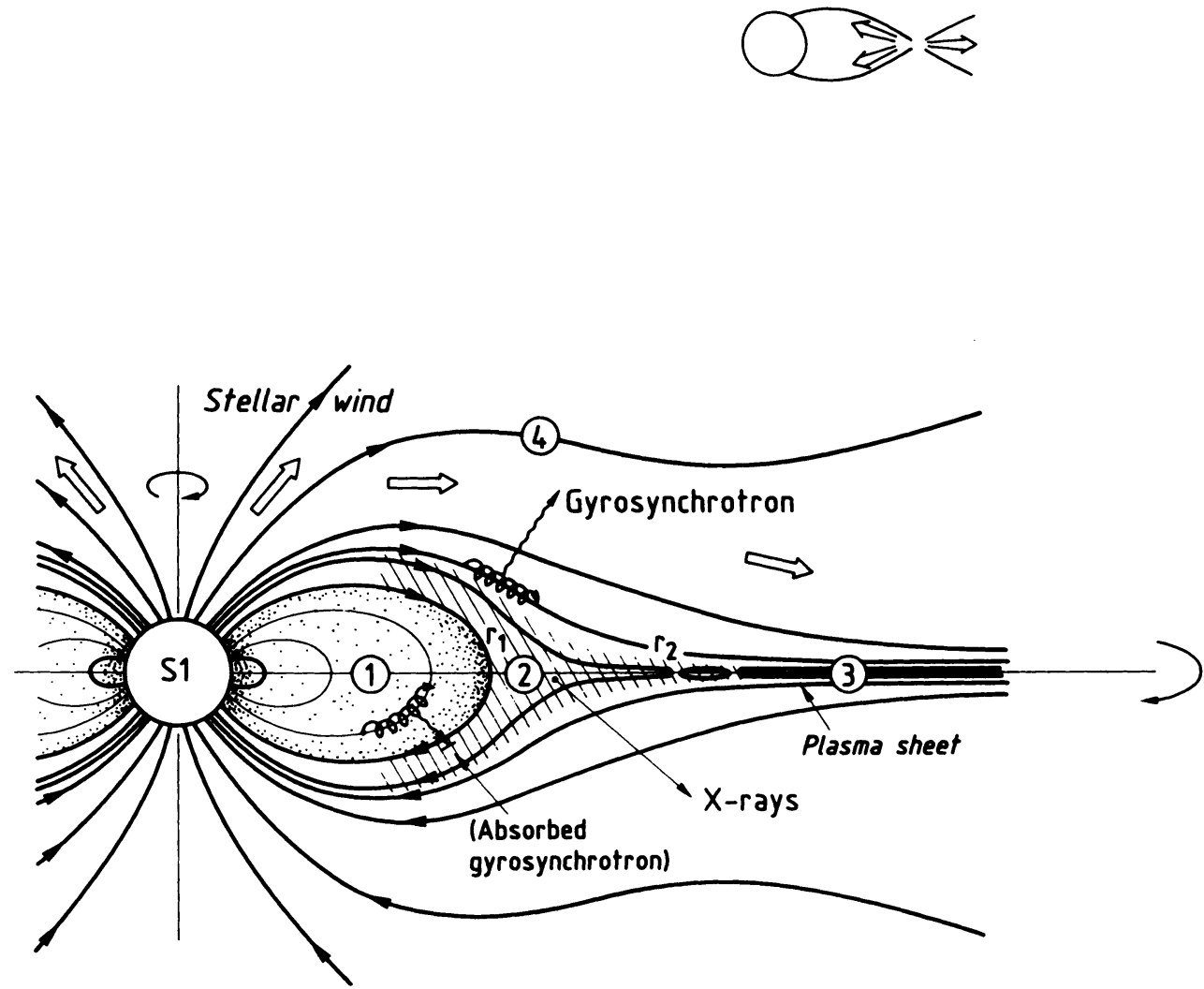

Fig. 7: Model of the magnetophere of the IR source S1 which may also be valid for HD 37479, except for the axis of rotation which is off by $73^{\circ}$ and, the plasma sheet that trails in the latter star (after André et al). 


\section{REFERENCES:}

André, P., Montmerle, Th., Feigelson, E.D., Stine, P.C., Klein, K.-L., 1988, Ap. J. 335, 940

Barker, P.K., 1986, LAU Coll. No. 87, Hydrogen Deficient Stars and Related Objects, Hunger, K. et al. (eds.), Reidel, Dordrecht, p. 277

Bohlender, D.A., 1988, Thesis, University of Western Ontario, London, Ontario

Bohlender, D.A., Brown, D.N., Landstreet, J.D., Thompson, I.B., 1987, Ap. J. 323, 325

Bohlender, D.A., Landstreet, J.D., 1988, IAU Symp. 132, The Impact of Very High S/N Spectroscopy on Stellar Physics, Cayrel de Strobel, G. and Spite, M. (eds.), p. 309

Drake, S.A., Abbott, D.C., Bastian, D.S., Bieging, J.H., Churchwell, E., Dulk, G., Linsky, J.L., 1987, Ap. J. 322, 902

Drilling, J.S., Hill, P.W., 1986, IAU Coll. No. 87, Hydrogen Deficient Stars and Related Objects, Hunger, K. et al. (eds.), Reidel, Dordrecht, p. 499

Groote, D., Hunger, K., 1982, Astr. Ap. 116, 64

Hamann, W.-R., 1981, private communication

Havnes, O., Goerth, C.K., 1984, Astr. Ap. 138, 421

Hunger, K., 1968a, IAU Coll. No. 87, Hydrogen Deficient Stars and Related Objects, Hunger, K. et al. (eds.), Reidel, Dordrecht, p. 261

Hunger, K., 1986b, IAU Coll. No. 90, Upper Main Sequence Stars with Anomalous Abundances, Cowley, C.R. et al. (eds.), Reidel, Dordrecht, p. 257

Hunger, K., Heber, U., Groote, D., 1989, Astr. Ap. 224, 57

Hunger, K., Heber, U., Groote, D., 1990, Properties of Hot Luminous Stars, Boulder-Munich Workshop, Germany, C.D. (ed.), p. 307

Iben, I. JR., 1965, Ap. J. 141, 993

Kaufmann, J.P., Schacht, P., 1975, Problems in Stellar Atmospheres and Envelopes, Baschek, B. et al. (eds.) Springer-Verlag Berlin, Heidelberg, New York, p. 80

Kurucz, R.L., 1979, Ap. J. Suppl. 40, 1

Lester, J.B., 1972, Ap. J. 178, 743

Paller, F., Stahler, S.W., 1990, The Birthline for Intermediate-Mass Stars, Submitted to Ap. J. Letters

Pauldrach, A., 1989, private communication

Phillips, R.B., Lestrade, J.-F., 1988, Bull. A.A.S. 20, 728 